\title{
Motor, linguistic, personal and social aspects of children with Down syndrome
}

\author{
Amanda Tragueta FERREIRA-VASQUES, Dionísia Aparecida Cusin LAMÔNICA
}

Department of Speech-Language Pathology and Audiology, Bauru School of Dentistry, University of São Paulo, Brazil.

Corresponding address: Dionísia Aparecida Cusin Lamônica - Al. Dr. Octávio Pinheiro Brisolla, 9-75 - Bauru - SP - Brazil - $17012-901$ - Phone: 55014 997717903 - 5501432358232 - e-mail: dionelam@uol.com.br

Submitted: March 20, 2015 - Modification: June 11, 2015 - Accepted: June 25, 2015

\section{ABSTRACT}

\begin{abstract}
A global developmental delay is expected from Down syndrome, affecting motor, cognitive, linguistic and personal-social skills. However, not always these delays are proportional; different conditions occur due to several intrinsic and extrinsic variables that must be controlled to form groups of greater homogeneity. Objective: To enhance personal-social, fine motor-adaptive, gross motor and linguistic skills among children with Down syndrome and compare them with typically developing children, matched for gender, socioeconomic status and mental age, while controlling some variables that interfere with the global development. Methods: The ethical aspects were fulfilled (Case No. 040/2009). The following inclusion criteria were considered: participants without a history of prematurity, very low birth weight, congenital hypothyroidism, significant hearing and vision problems, and signs of Autism Spectrum Disorder. After the inclusion criteria were considered, 40 children participated in the study, of which 20 had Down syndrome (experimental group - EG), these being of both genders and with chronological ages ranging from 38 to 63 months, and the other 20 being typically developing children (control group - CG), matching the EG in terms of gender, socioeconomic status and mental age, with this age ranging from 13 to 50 months. The evaluation consisted in applying the Denver Developmental Screening Test II, a test that assesses areas such as personal-social, fine motor-adaptive, linguistic and gross motor development. The results were subjected to statistical analysis using Student's t-test. Results: A statistically significant difference was verified between the groups for the language and fine motor-adaptive areas. Conclusion: Children with Down syndrome showed lower performance in language and fine motor skills when compared with typically developing children. There was no statistically significant difference in gross motor and personal-social areas. It is worth mentioning the importance of controlling the variables to deal with more homogeneous groups.
\end{abstract}

Keywords: Child. Down syndrome. Child language. Evaluation. Development.

\section{INTRODUCTION}

Down syndrome (DS) is a type of chromosomal abnormality that causes a range of physical, intellectual and clinical symptoms affecting individuals of different races, ethnic groups, and socioeconomic classes. Although the clinical presentation may vary, it is possible to predict structural alterations in the central nervous system, congenital heart disease, immunodeficiency, metabolic disorders, gastrointestinal problems, and also the characteristic physical phenotype, among others ${ }^{21}$.
It is striking due to global alterations in the development process, involving motor, language, cognitive, self-care and personal-social dimensions ${ }^{11}$, and it is associated with specific behavioral phenotypes ${ }^{26}$. The alterations found in different development areas are not always proportional, although each area influences and is influenced by one another $5,6,11,15,16,20,26-28$.

Alterations in motor skills are expected, mainly due to neuroanatomical and physiological changes that cause muscle hypotonia, alterations in primitive reflexes, joint hypermobility, and they also have a bearing on postural control and muscle synergy $y^{2,18,22}$. 
Such alterations directly interfere with exploitation of the environment, causing a reduction of crucial stimuli and experiences for the development of language, cognition and socialization ${ }^{22,29}$.

The cognitive development of children with DS seems to be characterized by differences between individuals ${ }^{26}$. Deficient cognitive skills, due to an intellectual problem, will directly influence the processing of information, thus causing alterations in attention, memory, language acquisition and other development skills ${ }^{4,7}$.

In the personal-social and self-care areas, the influence of family guidance, as well as the environment in which the children are inserted, is outstanding, considering the stimulation input they receive ${ }^{1,13,20,23}$ added to the opportunities for acting autonomously and independently throughout the different growth stages. The performance of children with DS tends to be backward in these areas.

The development of language occurs with delay and tends to follow the stages of typically developing children, despite the heterogeneity verified in the language performance of children with DS. Alterations in semantic, phonological, syntactic and pragmatic aspects are expected ${ }^{9,24,28,31}$. In this case, social development is closely related to the language development, and both are prone to environmental influence ${ }^{16}$. Although literature shows that one development area influences the other, there are gaps concerning the understanding of alterations and transformations that characterize these children's development ${ }^{26}$.

The literature shows that the performance of children with DS is not homogeneous $2,14,19,21,30$. This performance may be related to different variables that interfere with development such as, for instance, anatomophysiological conditions of the central nervous system, congenital hypothyroidism, hearing and vision losses, presence of behavior patterns in the autism spectrum, prematurity and low weight, among others.

Considering what has been mentioned, the objective of this study was to verify personal-social, fine motor-adaptive, gross motor and language skills of children with Down syndrome, and compare them with typically developing children matched for gender, socioeconomic status and mental age, controlling some variables that affect overall development such as congenital hypothyroidism, prematurity, low birth weight, significant hearing and vision problems and characteristics of the Autism Spectrum Disorder.

\section{METHODS}

Before its execution, this study was submitted for the appraisal and approval of the Research Ethics
Committee of the Institution where the study was conducted (Process No 040/2009).

The criteria for inclusion in the Experimental Group (EG) were the following: to have been diagnosed with Down syndrome, for trisomy 21, before the chronological age of three months; to have been born at term; to not have been born severely underweight; to have shown results indicative of normality in hearing and metabolism neonatal screenings (for congenital hypothyroidism); to have sight that does not hinder the accomplishment of the activities proposed; to have attended rehabilitation procedures since the first trimester of life and to be attending regular school.

The criteria for inclusion in the Control Group (CG) were the following: to have typical development (TD); to have been born at term; to have not been born severely underweight; to have results indicative of normality in hearing and metabolism neonatal screenings (for congenital hypothyroidism); to have vision skills that do not hinder the accomplishment of the activities proposed; to attend school; to be matched for gender, mental age and socioeconomic status with the EG.

The participants' mental age was established after evaluation through the New Version of the Stanford-Binet Method, adapted by Terman and Merril $^{25}$ (1960).

The Brazilian Criteria of Economic Classification (CCEB, 2011) 3 was used to check socioeconomic status. This instrument considers the family ownership of items (material assets) and the level of education of the person responsible for the family.

The Childhood Autism Rating Scale (CARS), of Brazilian standardization ${ }^{17}$, was used to verify the symptoms of Autism Spectrum Disorder.

After the inclusion criteria were applied, 40 children participated in the study, 20 with Down syndrome (Experimental Group - EG), of both genders and chronological age from three years and two months to six years (mean of four years and five months), and 20 typical developing children (Control Group - CG), matched to the EG for gender, socioeconomic status and mental age, aged from one year and one month to four years and two months (mean of two years and six months). Some children did not qualify for the inclusion criteria: in the EG, there were two children who had been born preterm, three children who had been born severely underweight, two with congenital hypothyroidism, one with signs indicative of Autism Spectrum Disorder, one who had failed in the neonatal hearing screening, two who did not attend regular school, and one who had initiated rehabilitation procedures after the sixth month of life.

After signing the Informed Consent Form, the legal responsible people answered an anamnesis 
protocol with information about the early life of the participant.

The birth weight of EG participants ranged from $1850 \mathrm{~g}$ to $3880 \mathrm{~g}$, with a mean of $2706 \mathrm{~g}$, while the weights of the CG participants ranged from $2500 \mathrm{~g}$ to $3850 \mathrm{~g}$, with a mean of $3187 \mathrm{~g}$. Regarding aspects of general health, as reported in the anamnesis, the EG participants showed one or more of the following health problems: heart disease $(30 \%)$, alterations to the stomach and duodenum (10\%), myopia (10\%) and jaundice (10\%). EG children that had myopia used corrective lenses. In the CG, no alterations were reported.

The studied participants were classified in classes A (10\%), B (60\%), and C (30\%) for the socioeconomic status.

Regarding the participant's education, the EG children were distributed in Nursery (50\%) and Kindergarten ( $50 \%$ ) classes, while the CG children attended Mini-Nursery (50\%), Nursery (45\%) and Kindergarten ( $5 \%$ ) classes.

The evaluation consisted in applying the Denver Development Screening Test $\mathrm{II}^{10}$ that considers four developmental areas, namely: personal-social, fine motor-adaptive, language and gross motor.

The statistical analysis was performed by applying the Student's $t$-test for each area of the Denver II test. The Student's $t$-test is a parametric statistical test used to compare the mean of two groups, applied in two independent samples.

\section{RESULTS}

\section{Characterization of casuistry}

In Figure 1, the profile of participants is characterized in terms of gender, chronological and mental age.

The steps and age of acquisition of the milestones of psychomotor development are shown in Figure 2.

In this casuistry, the acquisition of cervical spine equilibrium in the EG occurred at ages between 3 and 28 months, with a mean of 8.2 months; sitting without support occurred from the $7^{\text {th }}$ to the $38^{\text {th }}$ month of life, with a mean age of 15.1 months, and independent walking started at ages from 18 to 48 months, with a mean of 30.2 months. CG children fulfilled the development stages as expected for individuals with TD, i.e., the mean age for cervical equilibrium was 3.1 months; sitting without support, 6.9 months; and independent walking at 12.2 months.

Observing the age of the utterance of the first

\begin{tabular}{|c|c|c|c|c|}
\hline Pairs of participants & Gender & Chronological Age & Chronological Age & Mental Age \\
\hline & & CG (months) & EG (months) & (months) \\
\hline 1 & F & 22 & 43 & 21 \\
\hline 2 & F & 13 & 38 & 14 \\
\hline 3 & M & 28 & 51 & 29 \\
\hline 4 & M & 18 & 42 & 17 \\
\hline 5 & F & 43 & 46 & 41 \\
\hline 6 & F & 18 & 52 & 42 \\
\hline 7 & M & 44 & 60 & 41 \\
\hline 8 & M & 38 & 60 & 50 \\
\hline 9 & M & 50 & 62 & 37 \\
\hline 10 & M & 38 & 63 & 27 \\
\hline 11 & F & 26 & 44 & 41 \\
\hline 12 & M & 25 & 38 & 33 \\
\hline 13 & F & 41 & 50 & 26 \\
\hline 14 & F & 34 & 52 & 30 \\
\hline 15 & M & 26 & 66 & 20 \\
\hline 16 & M & 29 & 52 & 29 \\
\hline 17 & F & 19 & 41 & 29 \\
\hline 18 & M & 27 & 55 & 29 \\
\hline 19 & F & 28 & 72 & \\
\hline 20 & M & & & 42 \\
\hline
\end{tabular}

$\mathrm{F}=$ female; $\mathrm{M}=$ male; $\mathrm{CG}=$ control group; $\mathrm{EG}=$ experimental group

Figure 1- Distribution of participants in terms of gender, chronological age and mental age 
words, the EG participants showed this skill at ages ranging from 10 to 48 months, with a mean of 28.7 months, while in the CG this skill started at ages between 8 and 18 months, with a mean of 12.7 months.

The statistical analysis of the Denver II results revealed a statistically significant difference between the groups for language and fine motoradaptive areas.

Table 1 shows the mean and the standard deviation when comparing the CG and the EG performance in the application of the Denver II test

\begin{tabular}{|c|c|c|c|c|c|c|c|c|}
\hline \multirow{3}{*}{$\begin{array}{c}\text { Pairs of } \\
\text { Participants }\end{array}$} & \multicolumn{8}{|c|}{ Psychomotor Development } \\
\hline & \multicolumn{2}{|c|}{ CSE (months) } & \multicolumn{2}{|c|}{ sWS (months) } & \multicolumn{2}{|c|}{ IW (months) } & \multicolumn{2}{|c|}{ FW (months) } \\
\hline & CG & EG & CG & EG & CG & EG & CG & EG \\
\hline 1 & 2 & 4 & 6 & 9 & 11 & 27 & 10 & 18 \\
\hline 2 & 3 & 8 & 10 & 18 & 12 & 36 & 12 & 36 \\
\hline 3 & 4 & 6 & 7 & 9 & 12 & 32 & 18 & 34 \\
\hline 4 & 4 & 6 & 8 & 33 & 12 & --- & 12 & 36 \\
\hline 5 & 2 & 8 & 6 & 14 & 12 & 38 & 10 & 24 \\
\hline 6 & 5 & 5 & 7 & 8 & 13 & 27 & 16 & 40 \\
\hline 7 & 3 & 28 & 6 & 38 & 11 & 48 & 12 & 36 \\
\hline 8 & 3 & 3 & 7 & 7 & 12 & 27 & 24 & 13 \\
\hline 9 & 3 & 6 & 6 & 8 & 10 & 21 & 12 & 42 \\
\hline 10 & 3 & 5 & 9 & 9 & 12 & 23 & 11 & 10 \\
\hline 11 & 3 & 6 & 7 & 12 & 14 & 23 & 18 & 24 \\
\hline 12 & 3 & 4 & 6 & 7 & 14 & 18 & 8 & 36 \\
\hline 13 & 5 & 5 & 8 & 11 & 12 & 20 & 8 & 22 \\
\hline 14 & 3 & 11 & 8 & 13 & 13 & 22 & 11 & 24 \\
\hline 15 & 1 & 10 & 5 & 30 & 13 & 48 & 16 & 24 \\
\hline 16 & 4 & 5 & 6 & 9 & 13 & 20 & 12 & 30 \\
\hline 17 & 3 & 8 & 7 & 14 & 10 & 36 & 14 & 24 \\
\hline 18 & 3 & 9 & 7 & 12 & 14 & 20 & 12 & 24 \\
\hline 19 & 2 & 9 & 6 & 17 & 12 & 40 & 12 & 28 \\
\hline 20 & 3 & 18 & 6 & 24 & 11 & 48 & 12 & 48 \\
\hline
\end{tabular}

CSE=cervical spine equilibrium; SWS=sitting without support; IW=independent walking; FW=first words; CG=control

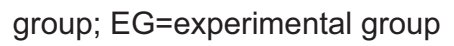

Figure 2- Age of acquisition of the milestones of psychomotor development

Table 1- Mean, standard deviation and p value resulting from the application of the Denver II test in the CG and EG

\begin{tabular}{ccccc}
\hline Denver II & Group & Mean & Standard Deviation & p value \\
\hline P-S & CG & 33.5 & 9.26 & 0.861 \\
& EG & 32.8 & 13.6 & $0.019^{*}$ \\
FG & GG & 35.4 & 10.3 & $0.034^{*}$ \\
& EG & 26.9 & 11.5 & 0.130 \\
\hline
\end{tabular}

$C G=$ control group; $E G=$ experimental group; $P-S=$ personal-social area; $L G=l a n g u a g e$ area; FMA=fine motor-adaptive area; $\mathrm{GM}=$ gross motor area; * $s$ tatistically significant difference with $p<0.05$ 
through the Student's $t$-test $(p \leq 0.05)$.

\section{DISCUSSION}

The sample of this study was designed to control important variables that have a bearing on child development, such as mental age, socioeconomic status, gender, birth at full term, birth weight, and absence of comorbidities, such as characteristics of the Autism Spectrum Disorder, metabolic alterations for congenital hypothyroidism and diagnosis of significant hearing and vision problems, which allowed a more homogeneous sample.

Prematurity is considered an important biological risk for development delay. The literature shows that preterms, mainly those with low weight, come into the world in a very marked condition of general weakness that usually puts them in a situation of risk, not only of survival, but also of possible shortcomings in terms of development ${ }^{8}$. According to the inclusion criteria, no child of the sample showed a history of prematurity.

Congenital hypothyroidism is frequent among individuals with DS. Indeed, thyroid hormones are vital for the development of the central nervous system, particularly during childhood, as they affect the neuronal migration and differentiation, the activation of the sympathetic nervous system, synthesis and secretion of neurotransmitters and the processes of myelination. Untreated hypothyroidism can intensify several symptoms associated with DS during childhood such as psychomotor development, somatic and intellectual growth ${ }^{19}$. Thus, the sample was controlled considering the range of variables interfering in the process of global development of children with DS.

Recent research studies show that children with DS can present characteristics that fulfill the diagnosis criteria for the Autism Spectrum Disorder ${ }^{30}$. The sample was controlled in such a way that no child included in the study presented characteristics of the spectrum associated with DS.

One factor considered of risk for the alteration of the development is the presence of congenital heart disease. However, this was not an exclusion criterion in this study, although authors did state that children with DS who presented congenital heart disease showed more difficulties to reach the motor milestones when compared with children with DS who did not have a history of these associated alterations ${ }^{2}$. Individually analyzing the performance of the six children with DS that showed heart disease, participating in this study, we verified that only one of them showed cervical equilibrium, independent walking and production of first words notably superior (older age in the acquisition of development milestone) than the mean of the EG.

Analyzing Table 1 , in the personal-social area, we observed no statistically significant difference between the groups, although the EG children had a chronological age superior to the CG. The personalsocial area has been considered the development area that change the least among children with DS $^{13,20,23}$, although it is not possible to establish these children's social profile as this skill depends on many variables that are usually found in the particular social environments they attend. Indeed, children with DS are seen as extremely sociable and affectionate, and they also have an easy temperament. However, although these individuals have fitted this stereotype, the temperament is by no means uniform ${ }^{23}$, since personal-social skills depend on the environment needs.

With effective social interactions and relationships initiated as early as possible, children tend to become more productive, showing the positive influences of such interactions and relationships that provide the social matrix for their overall development. The children who participated in this study started their intervention procedures still in the first trimester of life, and participated in an educational system from an early age, which may have contributed to these results.

The difference regarding the educational degree of the groups in this casuistry is noteworthy due to the chronological age, since the classes in the Brazilian educational system are formed considering chronological age rather than level of development and/or ability. This finding could represent different degrees of stimulation for children from different groups because of the specific curriculum for each educational program.

The language area was the most impaired when comparing the groups, even considering that this study has strict control over the variables. We emphasize that the language skills required in the Denver II test referred mainly to the expression of concepts and production of words.

In a previous study we affirm that expression skills are more impaired than the receptive ones in this population ${ }^{9}$, and that, regarding the cognitive profile, they present alterations varying from mild to severe. Language is the most extensively studied area in the context of the cognitive system, often giving a particular character to this performance considering the many involved variables ${ }^{31}$.

Regarding the performance in the fine motoradaptive skill, a statistically significant difference between the groups was found. In the Denver II test, this area verifies skills that include manual coordination, stimulus organization, accomplishment of delicate features, handling of small objects, involving, mainly, the cognitive and visual-motor skills. Studies show that the fine motor coordination is often impaired in these individuals ${ }^{6,22,29}$. The study by Santos, et al.22 
(2010) showed that the most impaired areas in the children with DS were language and fine motor co-ordination, thus supporting the findings of this study.

Specific coordination and postural problems have a considerable impact on the development of adaptive and functional skills, mainly in pre-school and school ages. Motor skills and acquisitions are determinant in social interactions, favoring the participation in interactional activities and in different social contexts in which language is developed ${ }^{29}$.

Qualitatively analyzing the sample, we verified that the EG children showed a delay in the neuropsychomotor development, observed from the acquisition of cervical equilibrium (mean of 8.2 months) to that of independent walking (mean of 30.3 months). The delay in the motor development of children with DS is expected, as well as the presence of hypotony, alteration of primitive reflexes and joint hypermobility that influence posture control, adequate muscle synergy, accomplishment of movements and motor coordination ${ }^{14,18}$.

The delay in the neuropsychomotor development is an indication that there are alterations in the acquisition of skills in other development areas ${ }^{14}$, mainly in the first years of life, as the movement is considered the basis for the learning of new skills. Through the motor action, the child has the possibility to explore spaces, objects and their relationships. Thus, the motor limitation may cause difficulties of interaction and manipulation of objects, with a loss of opportunities to develop the repertoire of learning experiences. The action creates and elicits cognition, through praxis, which has a form of mental planning that regulates, controls, integrates, elaborates and executes the child's intentions $^{12}$. In the comparison between the EG and the CG, there was no statistically significant difference in the gross motor skill. However, we must emphasize that these groups were matched considering mental age. Thus, the EG children showed chronological age superior to the CG children, which approximated the performance of these groups regarding the gross motor aspect. It is possible to affirm that children with DS showed an altered performance in this aspect because this performance was similar to the ones of younger children with TD.

Considering the difference of performance in the areas evaluated for each group, we noticed that children with DS showed better performance in the personal-social area, followed by the fine motor-adaptive and gross motor areas, and worse performance in the language area. A different behavior pattern was shown by children with TD, who showed a better performance in the fine motoradaptive area, followed by the language, gross motor and personal-social areas.

The literature shows that the heterogeneity in the performance of children with DS, and suggest longitudinal studies aimed to evaluate the process of language development within this population throughout the years $1,15,24,26$.

Reflecting on the methodological path that reached its peak with these results, the importance of studies that show a control of variables is emphasized as a way to get more homogeneous groups. On the other hand, the sample group is expected to be smaller with such control of variables. A restriction of this study to be emphasized is the absence of an analysis of the participants' central nervous system conditions by image exams, and the fact that individuals with congenital heart disease were not excluded.

In addition to the importance of matching of groups considering mental age, socioeconomic status and gender, as well as the control of variables considered as risk factors for development alterations, including premature birth, very low body weight, and congenital hypothyroidism, it is necessary to study these aspects while considering the anatomo-functional conditions of the nervous system of children with DS, for they would provide relevant information regarding the functioning of the processes that involve development. Longitudinal studies following the development of children with DS will be important to deepen the knowledge on this population development rhythm.

\section{CONCLUSION}

Children with DS showed an inferior performance in language and fine motor-adaptive skills when compared with typical developing children matched for mental age, gender and socioeconomic status. There was no statistically significant difference in the gross motor and personal-social areas.

\section{ACKNOWLEDGMENTS}

This study was supported by FAPESP - São Paulo Research Foundation, Process No. 2009/03355-9.

We would like to thank Professor José Roberto Pereira Lauris, professor of Statistics at the Bauru School of Dentistry, University of São Paulo, who helped us to carry out the statistical analysis of this study.

We also thank the participants and their families for their willingness to attend the interview and participate in the appraisal process. 


\section{REFERENCES}

1- Adamson LB, Bakeman R, Deckner DF, Nelson PB. Rating parent- child interactions: joint engagement, communication dynamics, and share topics in autism, Down syndrome, and typical development. J Autism Dev Disord. 2012;42:2622-35.

2- Anjos CC, Neto FG, Fontes VL. Comparative study of motor development of children with Down syndrome in relation to Denver II Scale. Fisioter Bras. 2008;9(6):387-91.

3- Brazilian Economic Classification Criteria. São Paulo: Brazilian Association of Research Companies (ABEP); 2011.

4- Campbell C, Landry O, Russo N, Flores H, Jacques S, Burack JA. Cognitive flexibility among Individuals with Down syndrome: assessing the influence of verbal and nonverbal abilities. Am J Intellect Dev Disabil. 2013;118(3):193-200.

5- Carney DP, Henry LA, Messer DJ, Danielsson H, Brown JH, Rönnberg J. Using developmental trajectories to examine verbal and visuospatial short-term memory development in children and adolescents with Williams and Down syndromes. Res Dev Disabil. 2013;34:3421-32.

6- Chen CC, Ringenbach SD, Albert A, Semken K. Fine motor control is related to cognitive control in adolescents with Down syndrome. Int J Disabil Dev Educ. 2014;61(1):6-15.

7- Edgin JO. Cognition in Down syndrome: a developmental cognitive neuroscience perspective. WIREs Cogn Sci. 2013;4:30717.

8- Fasolo M, D'Odorico L, Costantini A, Cassibba R. The influence of biological, social, and developmental factors on language acquisition in pre-term born children. Int J Speech Lang Pathol. 2010;12(6):461-71.

9- Ferreira AT; Lamônica DA. Comparing the lexicon of children with Down syndrome and typically developing the same mental age. Rev CEFAC. 2012;14(5):786-91.

10- Frankenburg WK, Doods J, Archer P, Bresnick B, Maschka $P$, Edelman N, et al. Denver II Training Manual. Denver: Denver Developmental Materials; 1992.

11- Freire RC, Melo SF, Hazin I, Lyra MC. Neurodevelopmental and relational aspects of baby with Down syndrome. Av Psicol Latinoam. 2014;32(2):247-59.

12- Hadders-Algra A. General movements: a window for early identification of children at high risk for developmental disorders. J Pediatrics. 2004;145(2 Suppl):S12-8.

13- Hippolyte L, Iglesias K, Van der Linden M, Barisnikov K. Social reasoning skills in adults with Down syndrome: the role of language, executive functions and socio-emotional behaviour. ] Intellect Disabil Res. 2010;54(8):714-26.

14- Malak R, Kotwicka M, Krawczyk-Wasielewska A, Mois E, Samborski W. Motor skills, cognitive development and balance functions of children with Down syndrome. Ann Agric Environ Med. 2013;20(4):803-6.

15- Martin GE, Losh M, Estigarribia B, Sideris J, Roberts J. Longitudinal profiles of expressive vocabulary, syntax and pragmatic language in boys with fragile-X syndrome or Down syndrome. Int J Lang Commun Disord. 2013;48(4):432-43.
16- Muluk NB, Bayoğlu B, Anlar B. Language development and affecting factors in 3- to 6-year-old children. Eur Arc Otorhinolaryngol. 2014;271(5):871-8.

17- Pereira A, Riesgo RS, Wagner MB. Childhood autism: translation and validation of the Childhood Autism Rating Scale for use in Brazil. J Pediat. 2008;84(6):487-94

18- Pereira K, Basso RP, Lindquist AR, Silva LG, Tudella E. Infants with Down syndrome: percentage and age for acquisition of gross motor skills. Res Dev Disabil. 2013;34(3):894-901.

19- Purdy IB, Singh N, Brown WL, Vangala S, Devaskar UP. Revisiting early hypothyroidism screening in infants with Down syndrome. J Perinatol. 2014;34(12):936-40.

20- Rihtman T, Tekuzener E, Parush S, Tenenbaum A, Bachrach SJ, Ornoy A. Are the cognitive functions of children with Down syndrome related to their participation? Dev Med Child Neurol. 2010;52(1):72-8.

21- Roizen NJ, Patterson D. Down's syndrome. Lancet. 2003;361(9365):1281-9.

22- Santos AP, Weiss SL, Almeida GM. Assessment and intervention in the motor development of a child with Down syndrome. Rev Bras Educ Esp. 2010;16(1):19-30.

23- Silva NL, Dessen MA. Síndrome de Down: etiologia, caracterização e impacto na família. Int Psicol. 2002;6(2):67-176. 24- Steele A, Scerif G, Cornish K, Karmiloff-Smith A. Learning to read in Williams syndrome and Down syndrome: syndromespecific precursors and developmental trajectories. J Child Psychol Psychiatry. 2013;54(7):754-62.

25- Terman LM, Merril MA. Stanford-Binet Intelligence Scale: manual for the third revision form. Boston: Pinneau; 1960.

26- Tsao R, Kindelberger C. Variability of cognitive development in children with Down syndrome: relevance of good reasons for using the cluster procedure. Res Dev Disabil. 2009;30:426-32. 27- Van Gameren-Oosterin HB, Fekkes M, Reijineveld SA, Oudesluys-Murphy AM, Verkerk PH, Van Wouwe JP, et al. Practical and social skills of 16-19-years-olds with Down syndrome: independence still far away. Res Dev Disabil. 2013;34(12):4599607.

28- Vicari S, Pontillo M, Armando M. Neurodevelopmental and psychiatric issues in Down's syndrome: assessment and intervention. Psychiatr Genet. 2013;23(3):95-107.

29- Volman MJ, Visser JJ, Lensvelt-Mulders GJ. Functional status in 5 to 7-year-old children with Down syndrome in relation to motor ability and performance mental ability. Disabil Rehabil. 2007;29(1):25-31.

30- Warner G, Moss J, Smith P, Howlin P. Autism characteristics and behavioural disturbances in $\sim 500$ children with Down's syndrome in England and Wales. Autism Res. 2014;7(4):433-41.

31- Ypsilanti A, Grouios G. Linguistic profile of individuals with Down syndrome: comparing the linguistic performance of three developmental disorders. Child Neuropsychol. 2008;14(1):148-70. 